\title{
Dilemas Ideológicos ante una Formación Universitaria con Sello Valórico Distintivo. El Caso de una Carrera de Ingeniería
}

\author{
Maite I. Jiménez, Jacqueline D. Reveco, María L. González y Carolina L. Astudillo \\ Pontificia Universidad Católica de Valparaíso, Escuela de Alimentos, Waddinton 716, Valparaíso-Chile. \\ (e-mail: mjimenez@ucv.cl, jreveco@ucv.cl, Igonzalez@ucv.cl, carolina.astudillo@ucv.cl)
}

Recibido Feb. 18, 2013; Aceptado Mar. 12, 2013; Versión final recibida May. 10, 2013

\begin{abstract}
Resumen
Se presenta un estudio sobre los espacios formativos relevantes en una universidad que declara tener un sello valórico distintivo. Se analizan aspectos relacionados con el rol formativo y si éste debiera ir más allá de la tecnificación profesional, para comprometerse más con una formación como personas. Para ello se realizó un estudio cualitativo de carácter interpretativo, en una perspectiva discursiva, mediante la identificación de los dilemas ideológicos. Como resultado se identificaron cinco dilemas en la formación valórica de los estudiantes y se reconocieron cinco espacios formativos relevantes para resolver futuros dilemas éticos. Se concluyó que la universidad ejerce un rol formativo a nivel valórico, orientando la reflexión crítica de lo que la sociedad les demandará como profesionales.
\end{abstract}

\section{Ideological dilemmas present in higher education with a declared explicit transcendent component. The case of an engineering major}

\begin{abstract}
This paper presents a study about the most relevant formative spaces in a university that claims to have a distinctive value-based seal. Aspects related to the formative role and if this should go beyond technical matters by giving a more humanistic formation. For this purpose, an interpretative qualitative study was carried out, identifying the ideological dilemmas of the students' focal groups taking part in this research. Results allowed the identification of five dilemmas in this transcendent formation and five formative spaces, to solve future professional ethical dilemmas. It is concluded that the university exerts a formative role of value-based issues guiding the critical analysis that society will demand from its future professionals.
\end{abstract}

Keywords: higher education, ethical dilemmas, moral values, transcendental formation 


\section{INTRODUCCIÓN}

\section{¿Qué es la Educación Superior en Chile?}

En Chile, la formación profesional, también llamada educación terciaria, está principalmente mediada por la oferta de la educación superior, conformada, según la ley 18.962 orgánica constitucional de enseñanza del año 1990, por centros de formación técnica, institutos profesionales, instituciones de fuerzas armadas y universidades.

Históricamente, ésta se perfiló como elitista y reservada a quienes dirigirían el futuro de la nación; sin embargo, luego de la reforma iniciada en 1980, su orientación ha sido hacia la masificación y la formación de capital humano que permita el desarrollo productivo del país (Robert, 2004). Lo anterior, ha provocado que esta etapa en la formación de la persona, sea vista con un carácter preferentemente tecnificante en el contexto de una profesión. De igual forma, el docente universitario, no precisa de una formación especializada en didáctica, y el uso y costumbre lleva a replicar los modelos ya naturalizados al interior de las instituciones (Chacin y Briceño, 2008).

En la última década, esta formación profesional ha sido objeto de amplio cuestionamiento, al ser sometido a procesos de evaluación externa. Reflejo de ello es la implementación de un sistema externo de aseguramiento de la calidad, como el instaurado a través de la creación de la comisión nacional de acreditación, con la ley 20.129 en el año 2006, que establece un sistema nacional de aseguramiento de la calidad de la educación superior. Como consecuencia a la irrupción de estos los procesos en la formación terciaria, que incorporaron requisitos de efectividad y eficiencia, se enfatizó aún más el anacronismo de los modelos tecnificados de formación profesional, en comparación a modelos que conciben la formación integral de la persona (Tünnermann, 2008).

\section{El Proceso de Bolognia y el Proceso Formativo}

En el contexto global, los cambios en Chile, fueron acompañados de un movimiento mundial hacia la armonización y la homologación entre universidades de diferentes países (Didou, 1996). El proceso de Bolognia puso en movimiento una serie de reformas a la educación Superior, focalizándose en el uso de estándares para comparar equivalencias entre instituciones (Smidt, 2010; Suchanek et al., 2012; Esen et al., 2012). Con ocasión del encuentro en Leuven en el 2009, la dimensión extra europea, alcanzó un nuevo carácter, bajo la noción de sociedad global del conocimiento (Hartmann, 2010), pero también se generó una mirada crítica de la educación superior donde el tradicional foco en la educación ha debido ceder su lugar a esquemas corporativos de trabajo (McArthur, 2011). Todo esto puso en tensión el concepto tecnificante de la formación profesional, para promover la internacionalización y la movilidad estudiantil. La noción de competencia se presentó como una alternativa para establecer las necesarias equivalencias, obligando a incorporar no solo aspectos procedimentales o cognitivos, sino también actitudinales (Canales y Schmal, 2012).

\section{La Calidad como un Proceso Evaluativo}

En Chile, la respuesta a estos movimientos internacionales dio paso a un proceso de aseguramiento de la calidad en la Educación Superior, cuestionando formalmente la calidad de las instituciones y de la formación entregada. Este hecho no ocurrió sólo en Chile, sino que estos procesos, organizados en un sistema de acreditación, comenzaron a extenderse desde Estados Unidos a todo el mundo, a partir de la década de 1980 (Fernandez, 2004).

Pese a ello, definir qué es calidad en educación superior, no tiene una única acepción (Harvey y Green, 1993; Vlãsceanu et al., 2007). La dificultad en consolidarla en un único concepto obedece a que está vinculada a un tipo de racionalidad (Baird, 2008) ya sea como un mínimo a lograr, como un atributo excepcional o como un estado de consistencia interna y externa; aunque en el caso de la educación superior, usualmente se enmarca como un ajuste a los propósitos - fitness for purpose - (Westerheijden 1999, Harvey y Newton 2004). Uno de los focos del sistema, es el alineamiento de los planes curriculares con los estándares requeridos para el ejercicio profesional (Harvey y Williams, 2010).

La evaluación de este cumplimiento se transforma en el eje de un proceso cuya aproximación ha sido preferentemente técnica, dejando de lado los aspectos éticos y generando una nueva cultura, la cultura de la rendición de cuentas -accountability- (Redón, 2009). 


\section{Competencias y Empleabilidad}

La Educación Superior, se ha convertido en un elemento vital para el desarrollo de una fuerza de trabajo productiva (Bardhan et al., 2013), donde los sistemas de medición de la calidad, frecuentemente demandan efectuar evaluaciones constantes para analizar la pertinencia del plan de estudios y la proyección de los ex alumnos en el ámbito laboral (Hernández et al., 2012). La continua pregunta acerca de la Empleabilidad, conlleva implícito entender que el perfil del graduado debe necesariamente constituirse por y para las necesidades laborales que demanda el mercado de trabajo (Vicente, 2012). Es así, que se convierte en uno de los estándares a los que debe enfocarse la formación terciaria (Ursin et al., 2010).

Diversos estudios, señalan que la obtención del primer trabajo, está fuertemente influenciado por características personales (Salas-Velasco, 2007; Tsiplakides, 2011). Se habla entonces de "competencias profesionales" para abarcar múltiples dimensiones, que la UNESO en su documento "La educación encierra un tesoro", estableció y que J. Delors (Molina, 2000) agrupó como: aprender a conocer, aprender a hacer, aprender a convivir y aprender a ser, en un intento por consolidar sus diferentes aristas.

Por otra parte, las políticas públicas, dirigidas por el Ministerio de Educación han orientado la renovación curricular hacia competencias. El acceso de las instituciones de educación superior a proyectos concursables y convenios de desempeño (MECESUP) ha financiado estos procesos, para lenta y progresivamente, incorporar la formación por competencias dentro del currículo en el contexto nacional. Es así que la docencia en las universidades se ha visto delimitada por una cultura evaluativa, lo que, unido a la incorporación de la noción de formación por competencias ha puesto en tela de juicio la forma de abordar la formación en valores o lo que ha venido a denominarse competencias transversales (Díaz y Márquez, 2007; Rodríguez et al., 2011; Siqueira, 2012). Este hecho hace necesaria una adaptación al interior de las aulas para ofrecer una formación más atingente a las transformaciones en los modelos de educación superior (Castaño y Garín, 2012).

En síntesis, la falta de reflexión institucional, en el rol formativo que ocurre durante el paso por las universidades, genera un profesional fragmentado, tecnificado y sin las herramientas para un actuar en forma integral. Entonces, se hace necesario explorar, de qué manera las instituciones están participando en la formación trascendente-valórica de sus estudiantes y cuáles son los espacios formativos que son reconocidos para tal efecto.

\section{MÉTODO}

\section{La Pregunta de Investigación}

La pregunta de investigación se convirtió en el eje de trabajo. La investigación realizada corresponde a una investigación situada, y como tal, demanda la voz del investigador quien se hace parte de la misma (Sisto, 2008). En un contexto donde la formación profesional se diseña desde un enfoque racional y positivista y a la vez es cuestionada acerca de su calidad y preparación para la vida profesional, permite la pregunta acerca de cómo se apropian los estudiantes de una formación valórica, declarada como eje de formación por la institución investigada, posibilitando la reflexión acerca de qué tensiones pueden aparecer y cómo son finalmente negociadas y resueltas por ellos durante su trayectoria formativa.

Es desde este cuestionamiento surgen las preguntas que guiaron los grupos focales: ¿Existe un sello valórico?, ¿Cómo se vuelve parte del proceso formativo? ¿Cómo los estudiantes participan de este proceso? y ¿Qué es lo que finalmente resulta del mismo?

Así, ellos fueron indagados sobre sus percepciones acerca de la carrera, de la universidad y si notaban alguna diferencia en la formación que estaban recibiendo, con respecto a lo que habría sido en otra institución, principalmente en cuanto a la formación en valores.

\section{Diseño del Estudio}

La presente investigación, fue de tipo cualitativa siguiendo las orientaciones de la Grounded Theory, (Glaser y Strauss, 1967), mediante caso instrumental (Stake, 1994), por cuanto éste sirve de escenario para la indagación. Se utiliza el análisis de discurso como marco de análisis, en el enfoque propuesto por Potter y Weatherell (1987), colocando el foco en los significados donde se concretan las relaciones sociales que dan origen a la experiencia subjetiva (Sisto, 2008).Es así que se realiza un muestreo teórico hasta la saturación de los datos, pues este tipo de muestreo permite producir aquellos datos pertinentes para que emerja la teoría (Charmaz, 2006). 


\section{Selección del Caso y Participantes}

El estudio se realizó en la Pontificia Universidad Católica de Valparaíso, una institución de educación superior que está entre las cinco mejores del país al que pertenece, posee una larga trayectoria como institución en el sistema nacional, abordando con destacada presencia los ámbitos de educación, investigación y gestión institucional. En la declaración de sus propósitos, destaca el reconocimiento al rol de la persona y promueve una visión valórica de hombre trascendente, en el marco del magisterio de la iglesia católica. Con carácter regional, ofrece formación de nivel de pre-grado y post-grado, llegando a la formación doctoral. Su distribución geográfica es dispersa, єeexistiendo siete campus. Académicamente, se organiza en facultades y éstas a la vez en unidades académicas, que son las que finalmente gestionan las carreras profesionales.

La carrera seleccionada, ofrece una formación en ingeniería con un diseño de cinco años para el título profesional. Posee la particularidad que no está alojada en la facultad de ingeniería, sino en otra más afín a su objeto de estudio. La unidad que la imparte, posee una trayectoria reconocida a nivel nacional en su campo disciplinar, ofreciendo formación a nivel pregrado y servicios profesionales a clientes externos. En un esfuerzo por mantener la confidencialidad, fueron modificadas las menciones a las otras universidades, incorporando el texto " $U$ ". Por otra parte, se omitieron los nombres de las carreras que dicta la universidad haciendo menciones a "Carrera 1", Carrera 2", etc. Además, debido a los diversos nombres que tienen los edificios y campus de la universidad objeto de estudio, se remplazaron dichos nombres por términos como "Locación 1", "Locación 2", etc.

\section{Producción de Datos}

La producción de datos, se realiza mediante la ejecución de grupos focales transcritos ad verbatim. Los participantes fueron estudiantes convocados a través de grupos focales. Estos se realizaron en dos bloques, uno para los estudiantes que se encontraban cursando los dos primeros años (Bloque inicial) y otro para los estudiantes que cursaban los dos últimos años de la carrera (Bloque final). Los grupos focales se realizaron en la siguiente secuencia: uno para el bloque inicial, uno para el bloque final; nuevamente uno para el bloque inicial y uno para el bloque final. Esta secuencia permitió la producción de los datos, completándose dos para el bloque inicial (20 estudiantes en total) y dos para el bloque final (14 estudiantes en total). Se finalizó el proceso mediante la discusión de los resultados con ambos bloques en una sesión plenaria.

\section{ANÁLISIS DE LOS DATOS}

Bajo el enfoque propuesto, la investigación reconoció en la voz de los participantes una conciencia analítica dirigida hacia su propia participación, así el proceso de producción de datos/análisis, se resuelve en un continuo inseparable, donde el analista debe ser capaz de contrastar los patrones encontrados, en las propias entrevistas (Sisto, 2008).

Para el análisis se trabajó en una perspectiva discursiva, identificando las posiciones de sujeto que surgen a partir de los dilemas ideológicos presentes en los discursos (Edley 2001). Los dilemas ideológicos, como herramienta de análisis, se refieren a los dilemas que se deben resolver desde una ideología "vivida", y como tales, las respuestas se construyen desde las creencias, valores y prácticas de una sociedad o cultura. Se caracterizan por la incoherencia, la fragmentación y la contradicción que presentan (Edley, 2001). Los argumentos compiten con los valores que la gente expresa al plantear estos dilemas. Es en la búsqueda de solucionar estos dilemas que los sujetos van tomando posiciones, que les permite situarse e interactuar al interior de una cultura (Reynolds et al., 2007).

\section{RESULTADOS Y DISCUSIÓN}

La institución reconoce en su declaración de principios "el esfuerzo institucional al servicio del pueblo de Dios y de la familia humana en su itinerario hacia aquel objetivo trascendente que da sentido a la vida" (Gómez, 1998). Es así, que su proyecto formativo declara el imperativo de generar espacios para cultivar y transmitir los valores inspirados en el Evangelio, en coherencia con el Magisterio. Su eje es impulsar la reflexión sobre el hombre y el sentido de responsabilidad en la construcción de la sociedad.

En la concreción de estos discursos institucionales, los participantes los "traducen" y re-interpretan para darles un sentido que los vuelva coherentes para ellos, es así que van surgiendo nuevos significados donde con base en creencias, valores y prácticas de la sociedad o cultura se hacen vida en forma de dilemas (Edley, 2001), ya que reflejan las ideologías que emergen a partir de las narrativas públicas que los interpelan (Smith y Sparke, 2008). 
Es entonces cuando los discursos introducen las formas de cómo los sujetos va tomando posiciones a lo largo de la conversación, va permitiendo conocer cómo los propios participantes co-construyen el significado, y cómo los discursos dominantes entran en juego (Sabaj, 2008). Al ser consultados por el sello valórico, no contestan desde lo experiencial, sino desde un deber ser, se vislumbra ya una tensión entre elementos que esperan sean definidos por otro, pero que a la vez los define y posiciona.

“- ...y ¿cuándo ustedes escuchan la frase o las palabras sello valórico, qué creen o imaginan que significa? De alguna forma lo han introducido, pero ¿ustedes digan qué es lo primero que se les ocurre que es un sello valórico?

-¿En qué sentido?

-Si lo tuviéramos que definir.

-Si lo tuviéramos que definir yo creo que la universidad entera, que los profesionales tengan respeto a valores por ejemplo una persona respetuosa, responsable, no sé, amiga del medio ambiente, no sé, cosas por el estilo.

-Sello valórico es cómo que me diferencia más específicamente a mí de otras personas también, para mi sello valórico, yo soy como persona y él va a ser otra persona, o sea si nos vamos a deber al resto del mundo, a la industria, a la empresa, a compañeros, a los papas, a los profesores, eso considero yo".

Es desde las propias definiciones que los hablantes co-construyen significados comunes, y relaciones que van dando cuerpo a sus palabras (Butler, 1997) así entendido, el currículo se convierte en una práctica social, ya que es a través de una práctica discursiva, donde la determinación curricular se vuelve concreta en una forma y estructura específica, impactando y transformando al estudiante en la mixtura de sus propios proyectos sociales y la estructura y determinación curricular, iniciales (De Alba, 1994). La contraposición de significados, y las tensiones que ellos provocan, aportan una generatividad en los discursos, matizando elementos de igualdad, autoridad, libertad, individualidad y pertenencia a un grupo (Billig, 1988).

\section{Primer Dilema: La Relación Entre La Universidad y el Estudiante}

El primer dilema, aparece en la valoración y creencias asociadas acerca de la naturaleza "católica" declarada como parte misma de la institución. Así, mientras un estudiante de primer bloque se mantiene ajeno a este hecho y concentra su capacidad como una autogestión. Se resume en la idea "...Uno tiene que, en el fondo, forjarse su propio camino si te dan todas las herramientas para hacerlo".

"-También son buenos porque se preocupan de uno, de que si entiende y...a pesar de que si uno mete mucha bulla, desorden... los profesores hablan y ahí nomás pero no, son súper buenos los profesores, se preocupan mucho de uno.

-Entonces ¿Cómo podríamos caracterizar ese compromiso? Ustedes dicen que sienten un compromiso en ciertas relaciones: con los profesores, con los funcionarios, a través de Internet ¿Qué características tiene ese compromiso que ustedes mencionan?

-Características totalmente positivas hacia nosotros, y obviamente nosotros tenemos que responderle a la universidad porque... o sea más que a la universidad, a nosotros mismos porque la universidad nos da todas las...

\section{-Facilidades}

-Las facilidades para poder inscribir, poder estudiar, poder estar estudiando, que te den beca... los que están con beca, los que no están con beca. Uno tiene que, en el fondo, forjarse su propio camino si te dan todas las herramientas para hacerlo, y desde ya eso es muy positivo".

En contraposición a este espíritu, si bien el grupo del bloque final reconoce un aporte y crecimiento en los espacios curriculares que aportan la diversidad, también es crítico y se vuelve demandante de una postura institucional frente a la sociedad, aspecto que se refleja en la expresión "...a pesar de que... eh... la universidad se dice católica y que entrega valores (tos) o que intenta entregarlos, creo que su aporte a la sociedad es mínima".

"-Yo igual concuerdo un poco con lo que dice ella, con respecto a los valores a la vez que uno si los trae desde... desde el hogar, pero esos ramos, como los ramos generales a mi me han hecho conocer otros 
temas los cuales yo por ejemplo ignoraba. Yo por ejemplo estaba tomando un ramo en Bioética, el asunto del medio ambiente, el tratamiento de las aguas, todos esos temas que quizás uno acá no los ve, y no es malo tomar ramos que son ramos generales y compartir con otras carreras, porque así también uno analiza otras realidades de otros compañeros, caras nuevas, porque uno cuando tiene que presentar, dar una exposición, uno está enfrente de los compañeros, pero cuando lo tiene que hacer frente a cien personas y que uno jamás en la vida ha visto eso también crea personalidad y eso ayuda mucho a la entrevista de trabajo y de hecho yo también me he dado cuenta de eso mismo, yo antes era mucho más tímida y ahora... eh... siento que estando acá... eh... en la Universidad o tomando otros ramos o no sé, teniendo ya derecho a práctica creo que la personalidad de por si... eh... como que... me desenvolví un poco más, por eso a mi me ha servido bastante.

- Yo creo que... eh... a pesar de que... eh... la universidad se dice católica y que entrega valores (tos) o que intenta entregarlos, creo que su aporte a la sociedad es mínima, o sea, creo que los ramos generales podrían estar enfocados de otra manera para en verdad aportar a la sociedad, porque nosotros predicamos mucho en la sala, pero en la práctica nada, entonces yo creo que eso le hace falta a la Universidad, porque no cuesta nada armar un grupo de personas. Ya, si estamos hablando de moral, estamos viendo este tema, a mi no me importa mucho ver los salmos por ejemplo, yo eso lo puedo leer en cualquier parte, lo puedo entender, puedo buscar en internet que significa cada palabra, cada frase. A mi me gustaría saber como puedo practicar eso, o sea, no es que voy a estar practicando un evangelio sino llevarlo en realidad a la sociedad, porque si nos decimos universidad católica. Es obvio, yo tengo mis valores de la casa... eh... y nadie me los mueve, pero yo podría potenciar esos valores y sacarle el jugo aportando a la sociedad".

Este dilema va generando que el estudiante del primer bloque se posicione en una actitud crítica dependiente del juicio externo, ellos reportan lo que han oído de otras personas y desde allí hacen suyo un discurso. Esta mirada cambia para los estudiantes del bloque final, donde la postura crítica se aborda desde un juicio interno, el cual demanda, tensiona e interpela a la institución desde la base de la coherencia que ellos esperan que exista entre las prácticas rutinarias y el discurso escrito.

Se va entonces elaborando en el tiempo una identidad que se construye desde estos actos de habla, que van dando origen a una acción colectiva que permite la transformación en las relaciones sociales (Butler, 1997). Desde esta contradicción, el posicionamiento que van tomando los estudiantes generan una continuidad temporal en su proceso formativo que los va transformando (De Alba, 1994).

\section{Segundo Dilema: El Sentido de la Vida Universitaria}

El segundo dilema, surge en las creencias asociadas a la noción que la vida universitaria debe constituir para ellos. Para los estudiantes del primer bloque la universidad "se abre" a ellos como un lugar exótico y lleno de nuevas experiencias, valoran la diversidad tanto en las personas que conocen como en las opciones de actividades, expresan El siguiente extracto, deriva de la pregunta acerca de cómo creen ellos sería estudiar esa misma carrera profesional pero en otra institución.

\section{“-¿Aquí es más científico?}

-Sí.

-Yo lo vería más matemático, es como más ingeniería.

-Sí, no es tan "alimentos".

-Es como que te marca el hecho que tenga el nombre de ingeniería, te marca más lo matemático. En cambio, en otras universidades, ya, tiene el nombre de ingeniería pero es como más diversidad. Aquí como que en la Católica, yo siento que marca mucho lo que es matemático... a pesar de que la "U*" igual es como la "escuela de la ingeniería" pero... los cálculos... Y también tiene como un mix de cosas, tanto carreras optativas que marcan tu valor de persona; y la parte académica que es prácticamente la malla que a uno le dan".

Este "mix de cosas" al que hacen referencia, alcanza una dimensión en su formación de persona, lo que entra en contraposición con el discurso de los alumnos del bloque final frente a una pregunta similar, quienes se han tecnificado en su comprensión de la vida universitaria, la ven más funcional, centrada en lo procedimental del actuar profesional y priorizada por la exigencia académica. Ellos expresan que estas acciones fuera de las asignaturas propias de la malla curricular "es como tiempo más perdido". 
“-¿Cómo?

-Lo que pasa que esos ramos generales como dice... son obligatorios, a pesar de que son optativos te obligan a tomar dos.

-Sí.

-Y eso por ejemplo son cosas que de verdad debieran quedar para la gente que quiera aprender eso, por ejemplo a mi no me gusta y me rehúso a tomar esas cosas, entonces no...

- Es como tiempo más perdido, porque podría ocuparse ese tiempo, por ejemplo, para otros ramos que nos podrían servir para destacarnos con otras universidades, yo creo que voy a ir un poquito más abajo, más allá de los ramos, yo creo que los ramos de cultura y todas esas cosas, yo creo que va un tema que es una... encuentro yo, ser pontificia, o sea, le da otro nombre, otro estrato, qué sé yo, pero yo creo que la universidad si tiene que, mínimo, guiar a sus docentes, ¿Por qué? Porque se supone que en el marco de la universidad, una de las cosas es que te enseñen ética, que te enseñen moralidad, etc. Y que el profesor sea un ejemplo a seguir, en un camino que nosotros vamos a tomar, pero yo creo que en esta universidad no pasa eso; o sea, los profesores llegan a hacer clases y chao. Entonces yo creo sinceramente que la pontificia es solamente una chapa".

De este dilema van surgiendo dos posiciones, una hedónica enfocada en una formación experiencial donde la riqueza de las relaciones y la diversidad de las formas de interacción van formándolos, para avanzar hacia una tecnificada, más formal y estratégica donde los esfuerzos son racionalizados y orientados hacia el desempeño académico, restando valor a todo aquello que no vaya en este sentido.

Esta contraposición, más que anecdótica responde a la naturaleza disciplinar, marcada por un currículo rígido y estructurado que favorece un enfoque positivista y tecnificado (Castells et al., 2010). Desde el enfoque de J. Delors (Molina, 2000), el saber hacer va ganando terreno sobre el saber ser, mediado por un énfasis en la formación desde un currículo que privilegia práctica.

Martínez (2006) establece cinco ámbitos en los que es posible integrar acciones que favorezcan la formación en valores y el aprendizaje ético: el de los contenidos curriculares, el de la relación entre estudiantes y profesores, el de las formas de organización social de las tareas de aprendizaje, el de la cultura participativa e institucional y el de la implicación comunitaria del aprendizaje académico. Sin embargo, desde los hablantes, no resulta tan obvio que la universidad sea un lugar en el que se aprenda un conjunto de saberes éticos y ciudadanos.

\section{Tercer Dilema: Acerca de su Visibilidad en el Sistema}

El tercer dilema, se presenta en la inserción y reconocimiento con que los estudiantes se auto-califican en relación a la comunidad universitaria. Los alumnos del primer bloque se sienten considerados y fuertemente integrados a las rutinas institucionales, valoran un sistema que los ordena y regula.

“-¿Cómo es acá?

- Acá todo es ordenadito, todo secuencia de pasos...

- $\mathrm{Si}$, pasas de este ramo a este ramo...

- Por ejemplo, igual es diferente a otras universidades tradicionales. En la " $\mathrm{C}^{*}$, yo tenía que tomar los ramos como una semana antes de entrar a clases. Acá no, acá hay una preinscripción de lo que uno tiene que tomar y cuándo va a ser, los profesores y todo eso.

- ¿Y qué valor le dan ustedes a esa práctica, a esa práctica de orden, de preinscripción? ¿Por qué tiene valor para ustedes, qué les dice eso de la universidad?

- Es que uno se despreocupa, uno dice “ya, ¿y cuándo va a ser?”... y llega un correo además.

- Eso es súper bueno.

- Se ve la preocupación, la responsabilidad. 
La expresión "Todo lo que hacen, te llega correo" señala como ellos identifican un esfuerzo institucional por mantenerlos como integrantes activos en el sistema. Esta visión se contrapone con los últimos años de la carrera, donde el peso académico y una marcada autogestión de sus necesidades van marcando el discurso. Hay una percepción de precariedad en la relación que les produce inestabilidad. Ellos señalan "estamos como aislados".

“- Geográficamente estamos más lejos, quizá... yo creo que es un factor, que estamos lejos.

- Y que no somos como de las carreras más requeridas.

- Tampoco estamos tan lejos pero se ve, se siente.

- Se siente, a veces parece colegio, yo encuentro que es como un colegio que tiene cero relación con el mundo exterior, pero es como un problema de clase que tiene esta universidad o sea, siempre se ha luchado... esto está aislado, los chicos de la "Locación 3" también están aislados; ahora la "Locación 4" está también en la punta del cerro, entonces nunca... nunca... claro, si usted le pregunta a un estudiante de la "Carrera 2", que es como la carrera estrella de esta universidad, o de "Carrera 1" o "Carrera 3"... claro viva la universidad pero comparto lo que dice ella, aquí no pasa eso".

La tensión entre ambas miradas emerge entre, los estudiantes que se integran y hacen partícipes de la comunidad, frente a otros que se sienten marginados y limitados en su relación institucional. En una mirada discursiva, estas palabras van constituyendo actos que posicionan a los estudiantes del bloque final desde una precariedad en relación a los del bloque inicial (Butler, 1997). Si bien se reconoce el rol del grupo en la transformación curricular (Nuñez y Oyanedel, 2009) se interpreta desde una postura crítica en relación a su espacio social (Bourdieu, 1985; Bourdieu, 1989), donde "El currículum es un intento de comunicar los principios esenciales de una propuesta educativa de tal forma que quede abierto al escrutinio crítico y pueda ser traducida efectivamente a la práctica" (Stenhouse, 1984).

\section{Cuarto Dilema: El Ser y el Deber Ser Institucional}

El cuarto dilema, aparece en la interpretación que ellos hacen de un cierto "deber ser" institucional, ellos arrojan un conjunto de expectativas sobre lo que la institución representa, vale decir, hay un imaginario acerca de lo que se debiera y no se debiera aceptar dentro de la institución. Así los estudiantes de primer bloque, señalan un atributo de prestigio como relevante y propio de la institución ("ser pontificia... es reconocida mundialmente").

- Yo creo que más que todo eso, yo creo que también te inculca valores porque yo estaba en la " $U^{*}$ y no... era... todo daba lo mismo. Acá no, es súper ordenado a nivel administrativo: uno tiene una duda, va a cierta parte y le sacan de la duda a uno...y eso lo de los valores, me gustó mucho eso.

- El resto... en función de estas diferencias: el hecho que sea pontificia, el hecho que sea católica, el tema valórico ¿qué opina el resto?

- No sé, yo por lo menos vi una comparación de cada universidad donde sale así como... la investigación que han hecho, como los profesores que son.... infraestructura también, todo eso. Como que se encuentra dentro de las mejores.

- Sí.

- Como en tercer lugar, creo.

- A nivel nacional.

- Sí.

Este aspecto que parece desmoronarse en los últimos años, hay un juicio descalificativo e incluso de reproche acerca de expectativas que no se cumplieron ("Es más la chapa que tiene").

"- Es más la chapa que tiene la universidad. Nosotros tuvimos la oportunidad de analizar el proyecto educativo que se presentó hace un tiempo y claro, sonaba increíble, se veía maravilloso... me pongo la camiseta de la Católica, pero ninguno... de las cosas que leímos, por lo menos de las cosas que veía yo, no se veía reflejado nada en la realidad. Tienen la chapa porque eran puras palabras bonitas las que se dicen unidas por... no sé, por un hilo invisible, porque no habían tiempos ni plazos que establecieran... no sé, a tanto tiempo vamos a hacer tal cosa. En cambio, conozco proyectos educativos de otras universidades que 
son más estructurados "vamos a hacer esto de aquí a dos años" y si no se hace de aquí a dos años, piden disculpas y lo aplazan más, pero lo revisó alguien. Acá en ese sentido, son como más...

- Flexibles.

- Sí flexibles... quizá extremadamente humanistas... no sé hay un punto que no... no sé, eso era como nada concreto.

-OK, bien. ¿A alguien le gustaría agregar algún punto sobre este elemento, acerca de la diferencia entre esta universidad y otras?

- Yo creo que otra diferencia que existe... bueno, yo creo que alguien que estudia en una tradicional, siempre se va a comparar con una persona que estudie en universidad privada. Nosotros sí tenemos un nivel superior a la universidad privada por el hecho del sentido que le da esta universidad al estudiante y a la universidad, o sea a lo que voy es que igual forman con... o sea, nosotros nos esforzamos estudiando, nos cuesta; en cambio, claro, como decía ella, en otras universidades no pasa lo mismo. Pero ¿Qué universidad? o sea esta chapa tiene la universidad, es una universidad tradicional que fue fundada y tiene como 95 años, 96 años esta universidad, o sea esta universidad tiene una trayectoria y eso no me puedo negar ¿Se entiende?"

Este dilema, da origen a que mientras los estudiantes toman inicialmente una posición de empoderamiento, al sentirse partícipes de una institución con la cual ellos pueden ser reconocidos como "mejores", da paso a una posición de reproche e interpelación, donde se demanda el cumplimiento de ciertas promesas implícitas que no reconocen como cumplidas. Es desde sus discursos que van constituyendo y constituyéndose en una comunidad de hablantes (Butler, 1997) y a vez van estableciendo su identidad como sujetos en un proceso de continua transformación (Revilla, 2003).

\section{Quinto Dilema: La Formación como Apertura al Cambio}

El quinto dilema, aparece en la forma en que ellos viven los cambios en relación a su formación y a lo que ellos conciben como debiera ser esa formación. El estudiante del primer bloque, valora el proceso de innovación que está experimentando, hay una libertad que no había experimentado, los profesores se relacionan con ellos de una manera diferente y este cambio lo viven con una expectativa positiva ("iba mucha gente y lo que me parecía bueno también era... como libre").

"-Encuentro que, bueno, lo otro es la flexibilidad de horario... por ejemplo, yo sabía que en acondicionamiento físico, creo que se impartía, bueno... yo una vez fui... el año pasado y se impartía como tres veces a la semana, me parece, y habían distintos horarios, y creo que el último horario era como a las ocho, se terminaba como a las nueve y media, que obviamente, iba mucha gente, por que, por lo mismo, porque no alcanzaba a... durante el otro horario, que era como a las dos me parece y... iba mucha gente y lo que me parecía bueno también era... como libre, o sea, llegabas, te registrabas, que carrera, cédula y llegabas a hacer deporte, por ejemplo, acondicionamiento físico. Lo que por ejemplo, yo tenía también compañeras de otra universidad que me dice que hay como un cupo limitado, por ejemplo, ya, treinta personas para ese...ese curso y no hay más, o sea, saltan de alegría si les dan el cupo, y... pero aquí es distinto, por ejemplo también en un área de los deportes... eh... yo participé en primer año, fui a... a la selección de ajedrez que tiene la Universidad y... eh... me acogieron, todo bien, son puros hombres y como yo soy mujer me subestimaron, entonces como que también existía una... no es como rivalidad, pero una... de... hay una mujer, y como que las mujeres son malas (para jugar), pero... es como machismo, pero no tan malo, pero si... eh... hay como de... libertad de ir, puedes ir, no es como que estés luchando por un cupo para entrar y bueno, si vas a dejar asistir, es porque no puedes seguir yendo y no hay, tampoco te ponen ni un problema. Entonces, aparte de eso, es como que de todas las distintas áreas como para ayudarte a la formación en verdad, de como persona.

- Que es importante también.

-¿A qué te refieres? Con lo de la formación como persona.

- Bueno, por ejemplo, como lo que yo fui, conocer a otro tipo de personas, otra área, porque eran muchos niños, estudiaban leyes, otros arquitectura, otros escenografía... eh... más o menos escuchar su experiencia, porque les preguntaba, también... eh... como el aprendizaje que ellos tienen y... bueno eso te sirve para hacer vida social, que es muy importante yo creo para aprender a desenvolverse en cualquier parte que estemos a futuro, y lo que nos decían algunos profesores es que a futuro uno como profesional se va a enfrentar con otros profesionales, entonces la base sería que desde el mismo momento que uno 
empiece a estudiar, ya empezar a tratar con gente de diferentes áreas. Entonces es bueno que la Universidad Católica tenga esas actividades extra programáticas donde pueda asistir gente de todos lados sin importar lo que este estudiando o cual es su fuerte, porque el día de mañana vamos a trabajar con cualquier gente".

En los últimos años de la carrera, estas expectativas se han transformado, la relación con los profesores se presenta tensionada y esta libertad es re-interpretada como abandono. Los estudiantes señalan la falta de articulación entre ellos, el juicio es crítico y concentra el mayor efecto sobre su formación ("tenemos que quedarnos con la visión de... algunos profesores").

“-Pero eso, ¿por qué pasa? Porque son muy individualistas en la Escuela y a nadie le interesa compartir con otro, a nadie le interesa que quieran tener una idea en común y hacer algo por la Escuela, a nadie le interesa. Todos vienen a estudiar, notas y chao...

- Exacto, ahora estamos entrando en otro tema, pero de vuelta a la Escuela...

-Tengo una compañera aquí que levantó la mano, estaba esperando.

- Yo concuerdo con ella, con que los profesores aquí están súper ocupados, porque ellos no sólo son profesores sino que cada... todos hacen investigación, pero el problema aquí, es un problema de Escuela, porque ellos tienen muy pocos docentes. Un docente imparte tres o cuatro ramos aquí y eso no está bien, porque así nosotros tenemos que quedarnos con la visión de... algunos profesores, siendo que otras ingenierías tienen la visión de ¿Cuántos profesores hay en su carrera?

\section{- Eso es cierto.}

- Yo eso no lo... no lo encuentro que esté bien, porque en la diversidad está el potencial. Nosotros somos todos distintos, eso es cierto, yo he aprendido en la Universidad, llegué con una mentalidad y mis compañeros me han cambiado mi visión, siempre para bien, para compartir y para todo, pero si nosotros tuviéramos otro tipo de profesores nos vamos a topar con una mayor diversidad de profesores buenos y malos, pero aquí nos quedamos con unos poquitos, que son buenos profesionales (risa), pero que no trabajan en equipo".

Entre la libertad y la guía, el currículo va tomando forma no solo en una formación profesional, sino en una trayectoria personal que aborda cada estudiante (Vicente, 2012). Así, mientras el estudiante del primer bloque adopta una postura más ingenua y receptiva, el estudiante del bloque final adopta una más crítica y demandante, ofreciendo una mayor claridad en las prácticas que deben establecerse en la formación profesional. El lenguaje, en las conversaciones de los estudiantes va cumpliendo funciones (Potter y Weatherell, 1987) permitiendo encontrar puntos de encuentro e identificación entre ellos, permitiendo la gestión de sus identidades (Mandiola y Ascorra, 2010) y es desde los dilemas que emergen que se van estableciendo espacios significativos y significantes de estos mismos discursos.

Balduzzi (2011) proporciona elementos sobre las representaciones que los estudiantes hacen acerca de su formación y cómo elaboran una relación asimétrica entre ellos y el docente. Entonces, el espacio cobra un significado particular como un escenario activo de las formas en que los discursos son materializados en prácticas y relaciones (Bourdieu, 1985; Bourdieu, 1989), y los espacios que son demandados por los participantes son los siguientes:

Espacios de Ruptura; principalmente en asignaturas que confronten los dilemas de su profesión. Ellos señalan la necesidad de cambiar la manera de entender la realidad y conciben en estos espacios, la oportunidad de que los profesores movilicen las creencias que los lleven a enfrentarlos en su ejercicio profesional.

Espacios de Retribución; La percepción es de inmediatez, lo que produce una pérdida de la historia que buscan recuperar, en los testimonios de exalumnos, redes o incluso el mismo sistema de ayudantías.

Espacios de Horizontalidad; en la relación docente-alumno, que ellos lo reconocen y significan como necesario pero inexistente, buscan un diálogo más fluido y participativo, a través de asambleas pero también en los espacios cotidianos.

Espacios de Ejercicio; donde las actividades académicas permitan ejercitar elementos de su formación actitudinal. El entrenamiento en valores como respeto, tolerancia, diálogo, empatía, en las acciones académicas que impregnen el currículo. 
Espacios de Diversidad; la oportunidad de ser cuestionados y demandado y a su vez de cuestionar y demandar, va construyendo dinámicas de formación que los obliga a perder la naturalidad con que ciertas creencias son asumidas.

\section{CONCLUSIONES}

Reconociendo que la educación superior ha sido fuertemente transformada en el enfoque formativo que tiene, respecto a la pregunta sobre cómo se apropian los estudiantes de una formación valórica, se identificaron cinco dilemas ideológicos con los que los estudiantes van constituyendo sus discursos. Estos dilemas, les permiten una negociación y elaboración de su formación profesional en el ámbito del sello valórico propuesto por la misión institucional. Por lo tanto, los resultados permiten concluir que la institución ejerce un rol formativo a nivel valórico, que va marcando los discursos de los estudiantes de cara a sus expectativas en relación a las demandas que la sociedad les presentará en su futuro que hacer profesional.

Además, fueron identificados cinco espacios formativos, como lo son ruptura, retribución, horizontalidad, ejercicio y de diversidad, que son relevantes en cuanto al rol que juegan en su formación profesional de los estudiantes, abarcando más que el tradicional contenido que se entrega en el aula.

Para finalizar se espera que esta experiencia e información permitan enriquecer las formas de abordar y entender el currículo, cuando se propone la formación valórica como uno de los ejes formativos.

\section{AGRADECIMIENTOS}

A la Dirección de Investigación de la Pontificia Universidad Católica de Valparaíso, por financiar el proyecto de sello valórico: "Indagación de las formas en que se manifiesta el sello valórico como carácter distintivo del perfil profesional en la formación del ingeniero de alimentos".

\section{REFERENCIAS}

Balduzzi, M., Representaciones sociales de estudiantes universitarios y relación con el saber. Espacios en blanco-Series Indagaciones. 21(2), 183-218 (2011).

Bardhan, A., Hicks, D. y D. Jaffee. How responsive is higher education? The linkages between higher education and the labour market. Applied Economics. 45(10), 1239-1256 (2013).

Baird, J. Whose Quality Conventions? The Application of Convention Theory in Higher Education. Tertiary Education and Management. 14 (1), 67-79 (2008).

Billig, M., Condor S., Edwards D., Gane J., Middleton D. y A. Radley. Ideological Dilemmas: A Social Psychology of Everyday Thinking. Sage Publication, Londres, Inglaterra (1988).

Bourdieu, P., Social space and symbolic power. Sociological Theory. 71(1), 14-25 (1989).

Bourdieu, P., Social space and the genesis of group. Theory and Society. 14(6), 723-744 (1985).

Butler, J., Lenguaje, poder e identidad. Editorial Síntesis, Madrid, España (1997).

Canales, T. y R. Schmal, Trabajando con Pósteres: una Herramienta para el Desarrollo e Habilidades de Comunicación en la Educación de Pregrado. Formación Universitaria. 6(1), 41-52 (2013).

Castells, M., Arese, A., Albizzati, E. y G. Rossetti, Propuesta para la Enseñanza de la Ingeniería: Un Espacio Curricular Creado desde la Investigación-Acción. Formación Universitaria. 1(2), 9-16 (2008).

Castaño, E. y A. Garín, Incorporación de Personas Adultas a los Estudios Universitarios: Aprendizaje Permanente para Arquitectos Técnicos. Formación Universitaria. 5(3), 17-26 (2012).

Chacin, M. y M. Briceño, El profesor universitario y la integración de la didáctica en la enseñanza universitaria. Paradigma, 29 (1), 21-40 (2008).

Charmaz, K., Constructing Grounded Theory: A Practical Guide Through Qualitative Analysis. Sage Publication, California, USA (2006).

De Alba, A., Currículum: crisis, mito y perspectivas. Miño y Dávila Editores, Buenos Aires, Argentina (1994). 
Díaz, J. y J. Márquez, Formación por competencias para los programas directores. Investigación y Postgrado. 22 (1), 239-260 (2007).

Didou, S., Educación superior, identidad cultural y globalización: las experiencias europeas en la perspectiva mexicana. IZTALAPAPA, Revista de Ciencias Sociales y Humanidades. ISSN: 0185-4259 (en línea). 38, 191-202 (1996). http://148.206.53.230/revistasuam/iztapalapa/viewissue.php?id=45. Acceso: 9 de mayo 2013.

Edley, N., Analysing Masculinity: Interpretative repertories, ideological dilemmas and subject positions. In: Discourse as a data. A guide for Analysis. First edition, 189-228, Sage Publication, Londres, Inglaterra (2001).

Esen, O., Gürleyen, I. y A. Oğuş-Binatlı. Impact of the Bologna Process on Turkish higher education: the case of Izmir University of Economics. European Journal of Higher Education. 2 (2-3), 187-197 (2012).

Fernandez, N., La evaluación y la acreditación de la calidad de la educación superior en américa latina y el caribe situación, tendencias y perspectivas. IESALC-UNESCO, Buenos Aires, Argentina (2004).

Glaser, B y A., Strauss. The discovery of grounded theory: Strategies for qualitative research. Sociology Press, Chicago, USA (1967).

Gómez G., La Universidad a través del tiempo. $1^{\text {a }}$ edición, Universidad Iberoamericana. 278 p. México D.F., México, (1998).

Hartmann, E., The new research agenda in critical higher education studies. Globalization, Societies and Education. 8(2): 169-173 (2010).

Harvey, L. y J. Williams. Fifteen Years of Quality in Higher Education. Quality in Higher Education. 16(1): 336 (2010).

Harvey, L. y J. Newton. Transforming quality evaluation. Quality in Higher Education. 2(10): 149-165 (2004).

Harvey, L. y D. Green. Defining Quality. Assessment \& Evaluation in Higher Education. 18(1), 9-34, (1993).

Hernández, C.; Tavera, M.; Jiménez, M., Seguimiento de Egresados en Tres Programas de Maestría en una Escuela del Instituto Politécnico Nacional en México. Formación Universitaria. 5(2): 41-52 (2012).

Mandiola, M. y P. Ascorra, Chilean management education: Rhetoric of pragmatism, consumerism, individualism and elitism. Cadernos EBAPE.BR 8(2), 373-387 (2010).

Martínez, M. Educación y Ciudadanía. Revista Iberoamericana de la Educación. 42(1), 85-102 (2006).

Molina, A., La competencia profesional en el ingeniero del nuevo milenio. Revista Facultad de Ingeniería. 8 (2), 65-71 (2000).

McArthur J., Reconsidering the social and economic purposes of higher education. Higher Education Research \& Development. 30(6): 1-13 (2011).

Nuñez, P. y M. Oyanedel. La negociación temática en la co-construcción del conocimiento realizada por estudiantes universitarios. Revista Signos. 42(69): 51-70 (2009).

Potter, J. y Wetherell, M., Discourse and Social Psychology. Sage Publication, Londres, Inglaterra (1987).

Redón, S., Auto evaluación institucional y acreditación como aseguramiento de la calidad de la educación: implicancias teóricas y prácticas. Estudios pedagógicos. 35(2), 269-284 (2009).

Revilla J.C., Los anclajes de la identidad personal. Athenea. 4(1): 54-67 (2003).

Reynolds, J., Wetherell, M. y S. Taylor, Choice and chance: negotiating agency in narratives of singleness. Sociological Review. 55(2), 331-351 (2007).

Rodríguez M., Mena D. y C. Rubio, Competencias que pueden Desarrollarse en la Asignatura de Mecánica en el Currículo de Ingeniería. Formación Universitaria. 4 (4), 3-12 (2011). 
Robert, H., Intelectuales y Educación Superior en Chile: de la Independencia a la Democracia Transicional, 1810-2001. $1^{\text {a }}$ edición, 1, 39-58, Ediciones CESOC, Santiago, Chile (2004).

Sabaj O., Tipos lingüísticos de análisis del discurso $(\mathrm{ad})$ o un intento preliminar para un orden en el caos. Revista de lingüística teórica y aplicada. 46(2) 119-136 (2008).

Salas-Velasco, M., The transition from higher education to employment in Europe: The analysis of the time to obtain the first job. Higher Education. 54(3), 333-360 (2007).

Sisto, V., La investigación como una aventura de producción dialógica: la relación con el otro y los criterios de validación en la metodología cualitativa contemporánea. Psicoperspectivas. 7(1), 114-136 (2008).

Siqueira, I. Inclusion and Solidarity as Education Methodology. Journal of Educational and Social Research. 2(7), 75-81 (2012).

Stake, R., Case studies. In: Handbook of qualitative research. Denzin, N. K. \& Y.S Lincoln (Eds.) 236-247, Thousand Oaks, USA (1994).

Stenhouse, L., Investigación y Desarrollo Del Currículum. 320 p. Ediciones Morata, Madrid, España (1984).

Smidt, H., Dalnäs, U., Sjögren, M., Josefson, K., The two-year master degree - combining Bologna reforms and internationalisation in Sweden. A study of the introduction of the Bologna reforms as one aspect of internationalization. In: Internationalisation of European Higher Education - An EUA/ACA Handbook (2010).

Smith, B. y A. Sparke. Contrasting perspectives on narrating selves and identities: an invitation to dialogue. Qualitative research. 1(1): 5-35 (2008).

Suchanek, J., Pietzonka, M., Künzel, R. y T. Futterer. The impact of accreditation on the reform of study programmes in Germany. Higher Education Management and Policy. 24(7), 1-24 (2012).

Tünnermann C., La calidad de la educación superior y su acreditación: la experiencia centroamericana. Avaliação (Campinas). 13(2), 313-336 (2008).

Tsiplakides, L. Similar performance, but different choices: social class and higher education choice in Greece. Studies in Higher Education. 36(1), 89-102 (2011).

Ursin, J., Aittola, H., Henderson, Ch. y J. Välimaa. Is Education Getting Lost in University Mergers? Tertiary Education and Management. 16(4). 327-340 (2010).

Vicente, M. (2012) E., Educación y Trabajo en Ciencias de la Educación: Aportes desde el Estudio de las Trayectorias Profesionales de sus Graduados. Formación Universitaria. 5(6): 51-62. (2012).

Vlãsceanu, L., Grünberg, L., y D. Pârlea. Quality Assurance and Accreditation: A Glossary of Basic Terms and Definitions. UNESCO-CEPES. ISBN: 92-9069-186-7 (en línea) (2007). http://unesdoc.unesco.org/images/0013/001346/134621e.pdf Acceso: 31 de marzo 2013.

Westerheijden, D., Where are the quantum jumps in quality assurance? Higher Education. 38(1): 233-254 (1999). 
